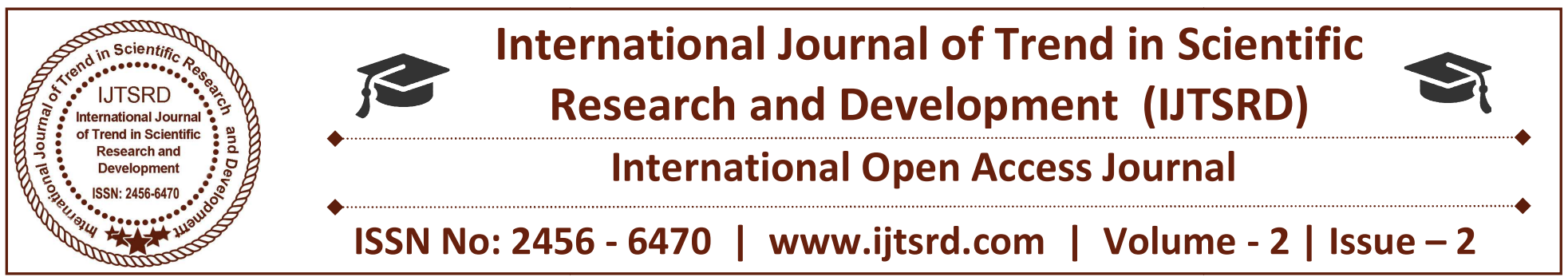

\title{
Overview of Various Industrial Fault Diagnosis Methods
}

\author{
Ms. Pranita A. Raut \\ M. Tech. Student, Department of Technology, \\ Shivaji University, Kolhapur, \\ Maharashtra, India
}

\author{
Dr. U. L. Bombale \\ Associate Professor, Department of Technology, \\ Shivaji University, Kolhapur, \\ Maharashtra, India
}

\section{ABSTRACT}

Fault detection plays an important role in high cost and safety critical processes. Early detection of faults can help to avoid normal event progression. Fault detection can be accomplished through various means. Fault diagnosis consists of all actions taken for fault recognizing, fault locating and root cause identification. For networking, Ethernet is suitable to act as the backbone to connect multiple domains and also for interworking within domains. Ethernet is one of the most common high speed interfaces found in homes and offices and there are some automobiles where Ethernet is being used to transport a variety of high speed data. Hence we can enhance a real time data driven algorithm for industrial fault diagnosis and localization of an Ethernet based systems. This paper presents a detail review of various fault diagnosis techniques, recent trends and their implementation for particular applications.

Keywords: Fault diagnosis, Ethernet, Engineering applications in industrial electronic equipments

\section{INTRODUCTION:}

Nowadays, industrial equipment and systems have become more complex and expensive, with less tolerance for performance degradation, productivity decrease, and safety hazards caused by unexpected faults, which stimulate an increasing demand for realtime fault diagnosis and fault-tolerant control techniques. Real time monitoring and fault diagnosis aim to detect, isolate, and identify any kinds of potential abnormalities and faults, so that necessary actions can be taken to avoid damage of any components, and even disastrous situations. As the scale and the complexity of the industrial system increases, the possibility of system suffering from interference increases. Defective hardware or loose connection of electronic equipments, and the ambient interference can produce intermittent faults in the system.

The intermittent connection fault is a common but hard troubleshooting problem. Fault is usually resulted from cable related problems in harsh working conditions, such as loose connectors or cable fatigue due to vibration or inappropriate maintenance. Thus in order to minimize the adverse effects of faults on the system performance and reliability, it is needed to effectively detect and accurately locate the faults in its early stage. Ethernet is the name of the most commonly used LAN today. Ethernet is a network protocol that controls how data is transmitted over a LAN. It is applicable to multiple domains because of its higher bandwidth. Ethernet networks possess one to one communication and hence it is necessary switched Ethernet networks. The bandwidth can be used efficiently than CAN and FlexRay. The key advantage to connecting over Ethernet lies in the overall savings in software development. There are various techniques by which we can use Ethernet for fault diagnosis system.

\section{LITERATURE SURVEY:}

Saugata S. Biswas, Anurag K. Srivastava and Dave Whitehead [2014] [1] presented the development of a new real time diagnosis algorithm that runs at a 
substation and continuously monitors the health condition of a circuit breaker trip coil assembly and suggests maintenance actions, if necessary. Developed algorithm detects the abnormalities, finds their root causes and also predicts the possibility of potential health problems for the circuit breaker trip coil assembly. Additionally, the monitoring architecture also allows remote access of data for engineering access.

Varun C. and Kathiresh M. [2014] [2] demonstrated the architecture of an Ethernet prototype platform for On-Board Diagnostic System (OBD) and a camera based application using an ARM centred system. Driver is also provided with a user interface through which user can read any of the sensor values by selecting on the list. Diagnosis of faults in parameters, notification of sudden changes to the driver, and indication regarding the reason for the fault are included in the system setup. The driver can find out the faults in car on the spot and this does not require an OBD scan tool for it. If in case the vehicle got struck in remote areas, the proposed OBD system inside the vehicle can be connected to the vehicle service centre by providing internet facility.

Leiming Zhang, Yong Lei and Qing Chang [2016] [3] proposed a novel IC fault localization algorithm for CAN based systems which utilizes the data link layer information of the CAN network for IC fault pattern analysis. Firstly, the fault events are defined by comparing the detected error records. Secondly, the context free grammar (CFG) is used to represent the IC faults based on network topology information. Then, based on the node error event pattern correlation, the concurrent localization/algorithm (CLA) is proposed to localize the local and backbone IC faults. Testbed is constructed and case studies are conducted to demonstrate the proposed methodology under various fault scenarios. Experiment results show that the IC fault locations identified by the proposed method agree well with the experiment setup.

Supriya Kelkar and Raj Kamal [2014] [4] proposed a new algorithm named adaptive fault diagnosis algorithm for CAN (AFDCAN). It is designed for low-cost resource-constrained distributed embedded systems. The proposed algorithm detects all faulty nodes on the CAN. It allows new node entry and reentry of repaired faulty nodes during a diagnostic cycle. AFDCAN is found to provide high fault tolerance and to ensure reliable communication. It uses single-channel communication deploying the bus-based standard CAN protocol. A hardware implementation of the proposed algorithm has been used to obtain the results. The results show that the proposed algorithm diagnoses all faults in the system. Analysis of the proposed algorithm proves that the algorithm uses a definite and bounded number of testing rounds and messages to complete one diagnostic cycle.

Bilal Akin, Seungdeog Choi, Umut Orguner and Hamid A. Toliyat [2011] [5] reported that the reference frame theory approach can successfully be applied to real-time fault diagnosis of electric machinery systems as a powerful toolbox to find the magnitude and phase quantities of fault signatures with good precision as well. The basic idea is to convert the associated fault signature to a dc quantity, followed by the computation of the signal's average in the fault reference frame to filter out the rest of the signal harmonics, i.e., its ac components. As a natural consequence of this, neither a notch filter nor a lowpass filter is required to eliminate fundamental component or noise content. Since the incipient fault mechanisms have been studied for a long time, the motor fault signature frequencies and fault models are very well-known. Therefore, ignoring all other components, the proposed method focuses only on certain fault signatures in the current spectrum depending on the examined motor fault.

Alberto Bellini, Fiorenzo Filippetti, Giovanni Franceschini, Carla Tassoni, Renzo Passaglia, Massimo Saottini, Giorgio Tontini, Mauro Giovannini and Alessandro Rossi [2002] [6] reported the experience gained by ENEL Produzione (previously the Italian Electric Board) on monitoring large induction motors cage condition. The diagnostic procedure is based on the motor current signature analysis and, in particular, on the two sideband current components near the frequency fundamental line that appear in the current power spectrum when a rotor bar/ring breakage occurs. According to the developed procedure, a diagnostic index obtained from these components is stored and its trend as a function of time allows for the detection of the occurrence of a failure in most cases. This event is clearly shown by the overcoming of a prefixed and triggered threshold. Moreover, machines with particular rotor magnetic structure are considered. In this case, unexpectedly high sideband components 
appear, even in the presence of healthy cages, and the test procedure was adapted to account for these conditions.

E.A.Mackenzie, J. Crossey, A. dePablo, and W. Ferguson [2010] [7] presented some of the recent developments in on-line monitoring and diagnostic techniques that help transformer operators to know the condition of their transformers and to make useful decisions on continuing operation, maintenance or replacement. It also illustrates how Dissolved Gas Analysis (DGA) is the heart of on-line monitoring as it is a well-established method of transformer diagnosis. Experience has shown that DGA can detect and give warning of $70 \%$ of the most common failures in power transformers. Some aspects of ongoing developments in DGA and its diagnosis are described.

\section{CONCLUSION:}

Online monitoring and diagnostics is useful tool to help operators to manage their assets and make decisions on continuous operation, maintenance or replacement. Early fault detection can increase the safety and reduce manufacturing costs. Number of issues must be considered when choosing particular fault detection method. Due to distributed generation and progression of industrialization, the fault levels are increased. This survey on fault diagnosis techniques provides us a better insight to choose and develop new techniques to obtain optimum accuracy in the diagnosis. Due to multimodalities available in fault diagnosis, obtaining high success rate and high diagnosing accuracy is prime important.

\section{REFERENCES:}

1. Saugata S. Biswas, Anurag K. Srivastava, and Dave Whitehead, "A Real Time Data-Driven Algorithm for Health Diagnosis and Prognosis of a Circuit Breaker Trip Assembly" IEEE transactions on industrial electronics 2014.

2. Varun C. and Kathiresh M., "Automotive Ethernet in On-Board Diagnosis (Over IP) \& In-Vehicle Networking" International Conference on Embedded Systems 2014.

3. Leiming Zhang, and Qing Chang, "Intermittent Connection Fault Diagnosis for CAN Using Data
Link Layer Information" IEEE Transactions on Industrial Electronics 2016.

4. Supriya Kelkar and Raj Kamal, "Adaptive Fault Diagnosis Algorithm for Controller Area Network" IEEE Transactions on Industrial Electronics, vol. 61, no. 10, October 2014.

5. Bilal Akin, Seungdeog Choi, Umut Orguner, and Hamid A. Toliyat, "A Simple Real-Time Fault Signature Monitoring Tool For Motor-DriveEmbedded Fault Diagnosis Systems" IEEE Transactions on Industrial Electronics 2011.

6. Alberto Bellini,Fiorenzo Filippetti, Giovanni Franceschini, Carla Tassoni, Renzo Passaglia, Massimo Saottini, Giorgio Tontini, Mauro Giovannini, and Alessandro Rossi, "On-Field Experience With Online Diagnosis of Large Induction Motors Cage Failures Using MCSA" IEEE Transactions on Industry applications 2002.

7. E. A. Mackenzie, J. Crossey, A. dePablo, W. Ferguson, "On-line Monitoring and Diagnostics For Power Transformers" Application to On-load Tap-changers 2010.

8. Kai Muller, Till Steinbach, Franz Korf, and Thomas C. Schmidt, "A Real-time Ethernet Prototype Platform for Automotive Applications" IEEE International Conference on Consumer Electronics 2011.

9. Shantanu Dutt, Vinay Verma, and Vishal Suthar, "Built-in-Self-Test of FPGAs With Provable Diagnosabilities and High Diagnostic Coverage With Application to Online Testing" IEEE Transactions on Computer-aided design of integrated circuits and systems 2008.

10. Z. Gao, H. Saxen, and C. Gao, "Special Section On Data-Driven Approaches For Complex Industrial Systems," IEEE Transactions on Industrial Information, 2013.

11. B. Mirafzal,"Survey Of Fault-Tolerance Techniques For Three-Phase Voltage Source Inverters," IEEE Trans. Ind. Electron., vol. 61, no. 10, pp. 5192-5202, Oct. 2014.

12. Z. Gao, C. Cecati, and S. X. Ding, “A Survey Of Fault Diagnosis And Fault-Tolerant Techniques- 
Part I: Fault Diagnosis With Model-Based And Signal-Based Approaches," IEEE Trans. Ind. Electron., vol. 62, no. 6, pp. 3757-3767, Jun. 2015.

13. I. Albizu, A. Tapia, J. R. Saenz, A. J. Mazon, I. Zamora, "Online Stator Winding Fault Diagnosis In Induction Generators For Renewable Generation," IEEE Electrotechnical Conference., pp. 1017-1020, May 2004.

14. A. Bouzida, O. Touhami, R. Ibtiouen, A. Belouchrani, M. Fadel, A. Rezzoug, "Fault Diagnosis in Industrial Induction Machines Through Discrete Wavelet Transform" IEEE Trans. Ind. Electron., vol. 58, no. 9, pp. 4385 4395, Sept 2011.
15. Andreas Kern and Thilo Streichert, "An Automated Data Structure Migration Concept From CAN to Ethernet/IP in Automotive Embedded Systems" (CAN over IP).

16. M. Rahmani, M. Pfannenstein, E. Steinbach,G. Giordano, and E. Biersack, "Wireless Media Streaming over IP-based In-Vehicle Networks.", IEEE International Conference on Communications Workshops 2009, pp. 1-6 June 2009.

17. M. Lee, H. Jin, "User-Level Network Protocol Stacks for Automotive Infotainment Systems", International Conference on Embedded and Ubiquitous Computing (ECU), 2010. 